\title{
Circulating miRNAs as Biomarkers of Acute Muscle Damage in Rats
}

\author{
Julien Siracusa, ${ }^{*}$ Nathalie Koulmann, ${ }^{* \dagger}$ Stéphanie Bourdon, ${ }^{*}$ Marie-Emmanuelle Goriot, ${ }^{\ddagger \S}$ and Sébastien Banzet ${ }^{\ddagger \S}$ \\ From the Armed Forces Biomedical Research Institute, * Brétigny-sur-Orge; the Armed Forces Biomedical Research Institute/Armed Forces Blood \\ Transfusion Center Jean Julliard, ${ }^{\ddagger}$ Clamart; the Ecole du Val-de-Grâce, ${ }^{\dagger}$ Paris; and INSERM U 1197, ${ }^{\S}$ Clamart, France
}

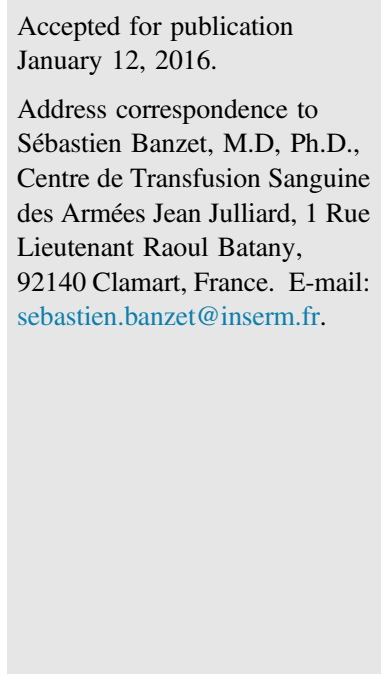

Accepted for publication January 12,2016

Address correspondence to Sébastien Banzet, M.D, Ph.D., Centre de Transfusion Sanguine des Armées Jean Julliard, 1 Rue Lieutenant Raoul Batany, 92140 Clamart, France. E-mail sebastien.banzet@inserm.fr.

Skeletal muscle may be damaged in many situations, such as trauma, drug use, exposure to toxins, heatstroke, exercise, metabolic disorders, or infection, and rhabdomyolysis can have serious consequences, such as acute renal failure or coagulopathy. ${ }^{1,2}$ Because clinical symptoms are nonspecific and inconstantly present, laboratory tests are necessary to help physicians in the diagnosis. Serum creatine kinase (CK) activity is routinely used to monitor muscle damage, but many limitations that impinge CK interpretation have been reported. ${ }^{3}$ High interindividual variability in baseline values, due to differences in sex, body mass index, ethnicity, or training status, is consistently reported. ${ }^{3}$ Furthermore, variations in serum CK activity in response to exercise have been reported. ${ }^{3}$ Hence, there is an effort to find new blood biomarkers of muscle damage that could be both clinically relevant and useful for physiological or clinical research. ${ }^{4-6}$

Mature miRNAs are short noncoding RNAs involved in the regulation of gene expression after transcription. Primary transcripts are processed into hairpin precursors that are cleaved into $3^{\prime}(3 p)$ and $5^{\prime}(5 p)$ mature miRNAs. In the cytoplasm, miRNAs may bind to target mRNAs and block translation or induce mRNA enzymatic degradation, and then are involved in a variety of cellular processes. miRNAs are also detected in most biofluids including plasma/serum, bound to proteins, or encapsulated within various microvesicles. There is an increasing interest in using extracellular miRNAs as circulating biomarkers of disease or physiological adaptations. ${ }^{8-11}$ If a major part of the literature investigates circulating miRNAs as biomarkers of cancer or cardiac diseases, they have recently been proposed to be relevant markers of tissue damage. ${ }^{12}$ Many miRNAs are ubiquitously expressed within cell types and tissues, but tissue-specific or tissue-enriched miRNAs have been described and could be released or secreted into the

Supported by contract PDH-1-SMO-2-0603 from the French Direction Générale pour l'Armement.

Disclosures: None declared. 
extracellular compartment as a consequence of cell damage. ${ }^{12}$ Therefore, an increase in their circulating levels can be indicative of specific tissue damage. For example, several reports have indicated that circulating liver-enriched miR122 is a reliable biomarker of liver damage, or that cardiacenriched miR-208a or miR-1 is a biomarker of acute myocardial infarction. , $^{913,14}$

Some miRNAs (miR-1, -133a, -133b, -206, -208a, -208b, -486 , and -499) have been described as muscle-specific or muscle-enriched (cardiac and skeletal muscle), two of which are specifically found in skeletal muscle (miR-133b and -206). The expression of those myomiRs is controlled by muscle-specific transcription factors, and those myomiRs are involved in a variety of cellular processes, including satellite cell proliferation, differentiation, fiber-type specification, exercise adaptation, and control of muscle mass or regeneration. ${ }^{15}$ They also are involved or dysregulated in neuromuscular diseases, rhabdomyosarcoma, and muscular atrophy. ${ }^{16-18}$ More interestingly, elevated myomiRs plasma/ serum levels are consistently found in patients with Duchenne and Becker diseases, and in animal models such as $m d x$ mice and dogs with golden retriever muscular dystrophy. ${ }^{19-21}$ They have therefore been proposed as biomarkers of muscular dystrophy (dystromiRs). However, high levels of circulating dystromiRs are not found in other dystrophies, such as Ullrich muscular dystrophy ${ }^{22}$ or myotonic dystrophy type $1 .^{23}$ Therefore, whether elevated plasma miRNA levels are due to myofiber damage or to specific mechanisms in dystrophin-related dystrophies is unclear. Roberts et $\mathrm{al}^{24}$ proposed that the very different expression patterns of miRNAs between skeletal muscle and plasma in $m d x$ mice might result from a specific biological response rather than a leak due to impaired sarcolemmal integrity.

We previously provided arguments to propose that myomiRs could be biomarkers of muscle damage in healthy organisms. ${ }^{25}$ We compared plasma levels of muscle-specific miRNAs in response to eccentric (damaging) and concentric (nondamaging) exercise in humans, and found that miR-1, $-133 a,-133 b$, and $-208 b$ were increased after eccentric exercise only. However, other studies have proposed that they could be mediators or biomarkers of skeletal muscle adaptation to exercise rather than biomarkers of muscle damage. . $^{10,11,26,27}$

Many studies in the literature have explored circulating miRNAs as biomarkers of diseases, but discordant and questionable results have been reported. ${ }^{28}$ It is now clear that a strong methodological approach is needed to identify and validate miRNA biomarkers. ${ }^{28,29}$ Our aim was to explore changes in circulating miRNAs in response to acute muscle damage in healthy organisms. We used a welldescribed model of myotoxic muscle damage in rats to identify new biomarker candidates. We widely characterized the response of our biomarker candidates (kinetics, amplitude, and proportionality to damage extent), as well as the influence of factors that could affect their circulating levels (sex, age, muscle phenotype, hemolysis, and platelet contamination or activation).

\section{Materials and Methods}

\section{Animals}

Young ( 2 months) male or female and aged (13 months) male Wistar rats were purchased from Janvier Labs (LeGenest-Saint-Isle, France). All experiments were performed in accordance with the Declaration of Helsinki and received prior approval from the local animal ethics committee.

\section{Experimental Design}

In experiment 1 , circulating miRNA profiling, 72 young male rats were randomly assigned to receive notexin injection in soleus muscle (NTX; $n=32$ ) or sham operation (SHAM; $n=32$ ) or to the control group (CON; $n=8$ ), and were sacrificed at $6,12,24$, or 48 hours after muscle damage.

In experiment 2 , influence of sex and age on circulating miRNAs, 24 female $(\mathrm{F})$ and 24 aged $(\mathrm{A})$ rats were assigned to receive notexin injection in soleus muscle (F-NTX, $n=8$; A-NTX, $n=8$ ) or sham operation (F-SHAM, $n=8$; A-SHAM, $n=8$ ) or to a control group (F-CON, $n=8$; A-CON, $n=8$ ), and blood was collected at 12 hours after muscle damage.

In experiment 3, influence of muscle phenotype on circulating miRNAs, eight young male rats were injected with notexin in the fast-type extensor digitorum longus muscle (EDL), and sacrificed 12 hours later. Results were compared to those obtained at 12 hours after soleus injury in experiment 1. Plantaris muscles were also collected for single fiber analysis.

In experiment 4 , influence of the extent of muscle injury on circulating miRNAs, notexin was injected into two EDL muscles ( $2 \mathrm{EDL} ; n=8$ ) or two EDL + two tibialis anterior muscles $(2 \mathrm{EDL}+2 \mathrm{TA} ; n=6)$. Blood and muscles were collected 12 hours later and data were compared to the findings from the EDL group in experiment 3 (1 EDL; $n=8)$.

In experiment 5 , influence of pre-analytical conditions, red blood cells (RBCs) and platelets were isolated from CON rats from experiment 1 and were used to enrich control plasma pools from these animals. Plasma samples from five CON rats and four NTX rats at 12 hours from experiment 1 were used to separate the vesicular and nonvesicular fractions.

\section{Surgical Procedures and Tissue Sampling}

Rats were anesthetized with an i.p. injection of a mixture of $60 \mathrm{mg} / \mathrm{kg}$ of ketamine (Laboratoire Renaudin, Itxassou, France) and $0.5 \mathrm{mg} / \mathrm{kg}$ of medetomidine (Elanco, Greenfield, IN). The muscle was surgically exposed, and $100 \mu \mathrm{L}$ (200 $\mu \mathrm{L}$ for aged male rats) of notexin $10 \mu \mathrm{g} / \mathrm{mL}$ (Latoxan, Valence, France) was injected either into soleus muscle (experiments 1 and 2), EDL muscle (experiments 3 and 4), or TA muscle (experiment 4). The SHAM groups were 
subjected to the same surgical gesture, with no muscle damage. Pain after surgery was prevented by an s.c. injection of $0.05 \mathrm{mg} / \mathrm{kg}$ of buprenorphine (Axience, Pantin, France).

Prior to the sacrifice, rats were anesthetized with an i.p. injection of $150 \mathrm{mg} / \mathrm{kg}$ of pentobarbital sodium (Ceva Santé Animale, Libourne, France). Anesthetized rats were sacrificed by exsanguination, and whole blood was drawn into a $\mathrm{K}_{2}$ EDTA tube (BD Vacutainer; Becton, Dickinson and Company, Plymouth, UK) for miRNA analysis, or into a lithium heparin tube (BD Vacutainer; Becton, Dickinson and Company) for CK measurement. Soleus, EDL, and TA muscles were harvested and frozen in liquid nitrogen. Plantaris muscles were placed in RNAlater (Ambion, Austin, TX) and stored at $-20^{\circ} \mathrm{C}$.

\section{Plasma miRNA Extraction}

The blood was centrifuged $\left(2000 \times g\right.$ for 10 minutes at $\left.4^{\circ} \mathrm{C}\right)$. The plasma was transferred to a new vial and centrifuged again. Total RNA was isolated from $100 \mu \mathrm{L}$ of plasma with a mirVana PARIS Kit (Ambion), with an additional precipitation step: Column elution was performed, with $180 \mu \mathrm{L}$ of sterile water, $18 \mu \mathrm{L}$ of sodium acetate $3 \mathrm{~mol} / \mathrm{L}$ (SigmaAldrich), $396 \mu \mathrm{L}$ of ethanol (100\%), and $1 \mu \mathrm{L}$ of GlycoBlue (Ambion) added. RNAs were precipitated for 20 minutes at $-20^{\circ} \mathrm{C}$. After centrifugation $(12,000 \times g$ for 15 minutes at $4{ }^{\circ} \mathrm{C}$ ), the pellet was washed with $70 \%$ ethanol, dried, and resuspended in $12 \mu \mathrm{L}$ of sterile water.

\section{cDNA Synthesis}

cDNA was synthesized from $5 \mu \mathrm{L}$ of total RNA diluted 1:6 in a $10-\mu \mathrm{L}$ reaction volume using the Universal cDNA Synthesis Kit (Exiqon, Vedbaek, Denmark) according to the manufacturer's protocol.

\section{Circulating miRNA Profiling (Experiment 1)}

All real-time quantitative (qPCR) assays were performed on a LightCycler 480 instrument (Roche Applied Science, Mannheim, Germany). First, Rattus norvegicus (rno)-miR-103a-3p was used for each sample as a quality control, with a quantification cycle cutoff of $\leq 32$. Then data on cDNAs in the CON and NTX groups were pooled for the 6-, 12-, and 24-hour time points. A total of 752 miRNAs were profiled in each pool with microRNA Ready-to-Use PCR, Mouse\&Rat panel I+II, V3.M (Exiqon) according to the manufacturer's protocol. Seventy-two miRNAs were selected based on detectability (quantification cycles, $\leq 35$ ) and variations in response to muscle damage (fold-change, $\geq 1.5$ ). Other miRNAs were selected based on the literature (rno-miR-126a-3p and rnomiR-208a-3p) as well as potent reference miRNAs.

Two different primer pairs for the rno-miR-208b-3b sequence (miRBase release 21, http://www.mirbase.org, last accessed January 10, 2016) were designed, but no signal was amplified. As the profiling included Mus musculus (mmu)-miR-208b-3p primers that consistently amplified a product with a profile similar to those of muscle-specific miRNAs, we compared the two sequences and found that the rno sequence had 4 bases missing at the $5 \mathrm{p}$ end. An alignment of 10 mammalian sequences of miR-208b-3b showed $100 \%$ conservation among species (data not shown). Alignment of the pre-miRNA sequences (mmu- and rno-mir-208b) demonstrated that the missing 4 bases UUGU are present on the rat transcript (data not shown). Therefore, we believe that the miRBase rat sequence may be incomplete, and in the present work, all measurements of miR208b-3p were performed with mmu primers.

Customized qPCR plates, Pick-\&-Mix microRNA PCR panels (Exiqon), were designed for the 72 selected miRNAs. Briefly, $500 \mu \mathrm{L}$ of $2 X$ PCR Master Mix were combined with $493.75 \mu \mathrm{L}$ of RNase-free water and $6.25 \mu \mathrm{L}$ of cDNA; $10 \mu \mathrm{L}$ of this mixture was distributed into each well. For experiments 2 to 4, individual qPCR was achieved with Exiqon primers in a $10-\mu \mathrm{L}$ reaction volume, with $4 \mu \mathrm{L}$ of cDNA (diluted 1:80). The number of reference miRNAs and their stability were determined using geNorm version 3.4 (https://genorm.cmgg.be, last accessed 2006). ${ }^{30}$ Quantification was the geometric mean of the quantification performed with each reference miRNA. All miRNAs used as references in the experiments are reported in Supplemental Table S1.

\section{CK Measurement}

Plasma CK activity was measured using a Cobas Integra 800 analyzer (Roche Diagnostics, Meylan, France) following the manufacturer's protocol.

\section{Muscle Histological Examination}

Hematoxylin phloxine saffron staining was performed on $8-\mu \mathrm{m}$ cryosections of soleus or EDL muscle. Images were acquired with a Leica DMI6000 B microscope (Leica Microsystems, Wetzlar, Germany).

\section{Muscle Tissue miRNA Measurement}

Muscle RNA was extracted with a miRNeasy Mini Kit (Qiagen, Courtaboeuf, France) from 10 to $15 \mathrm{mg}$ of muscle homogenized in $700 \mu \mathrm{L}$ of QIAzol Lysis reagent (Qiagen) according to the manufacturer's protocol. Reverse transcription was performed with a Universal cDNA Synthesis Kit (Exiqon) from $5 \mu \mathrm{L}$ of diluted RNA (5 to $10 \mathrm{ng} / \mu \mathrm{L}$ ) as described in $c D N A$ Synthesis, and $4 \mu \mathrm{L}$ of cDNA (diluted 1:80) was used for PCR reactions.

\section{Single Fibers Analysis}

Single fibers were isolated under microscopy using sharpended tweezers and cut into two equal parts. One half was placed in $20 \mu \mathrm{L}$ of myosin extraction solution; myosin 


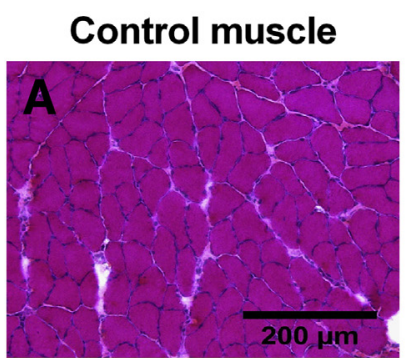

E
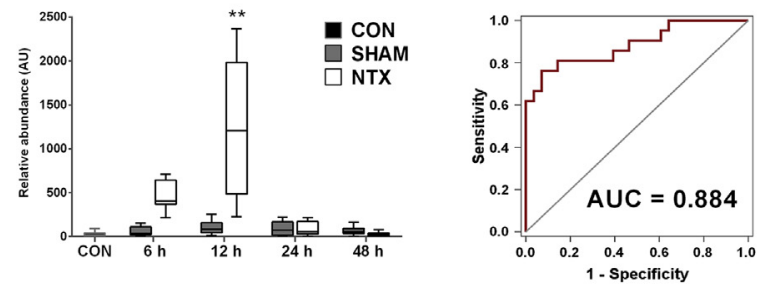

G

rno-miR-133b-3p
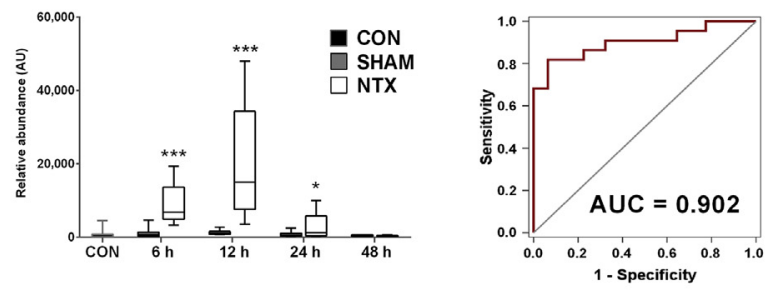

I mmu-miR-208b-3p
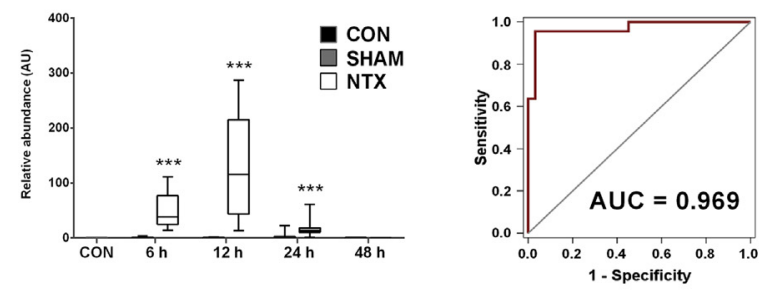

K rno-miR-378a-3p
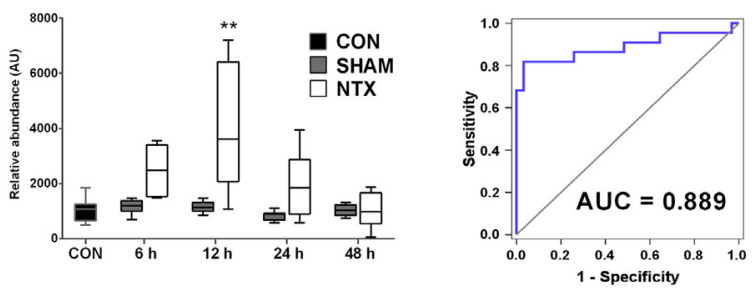

M

rno-miR-136-5p
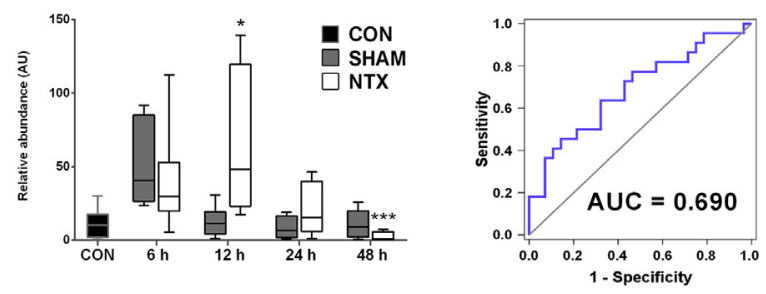
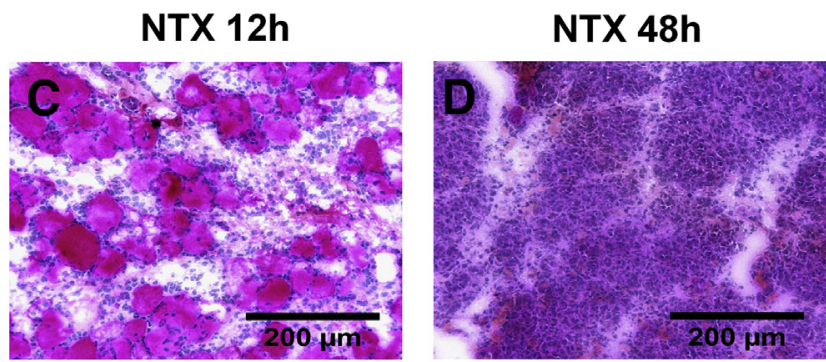

F

rno-miR-133a-3p
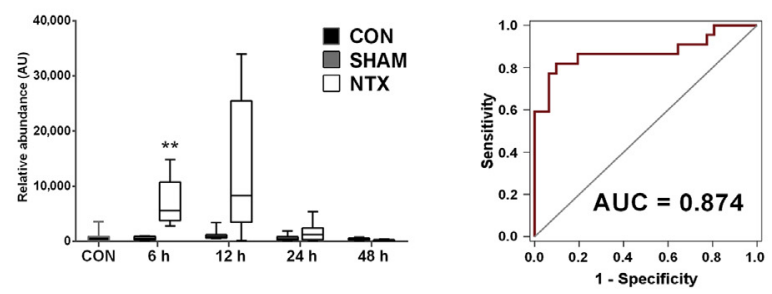

H

rno-miR-206-3p
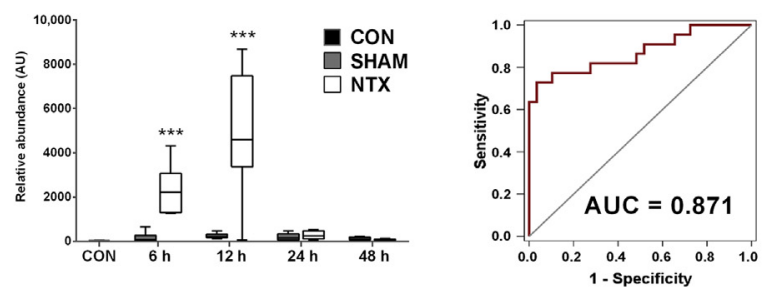

J

rno-miR-499-5p
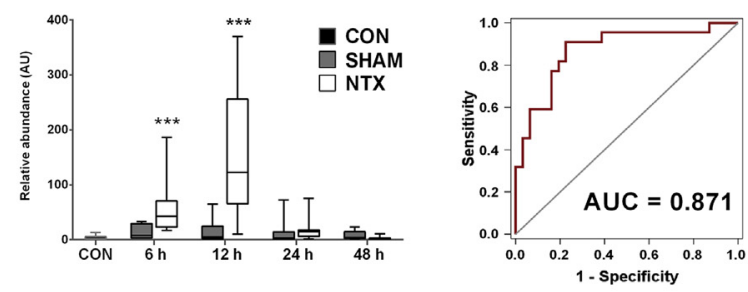

L

rno-miR-434-3p
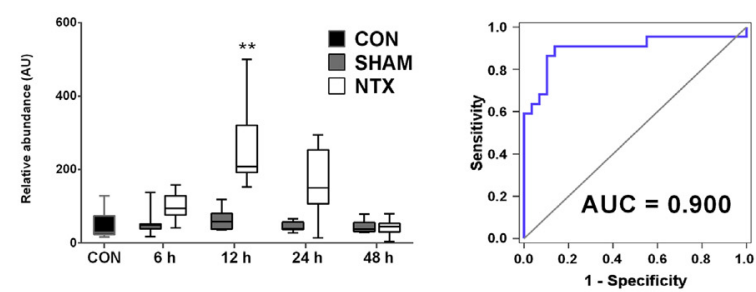

N
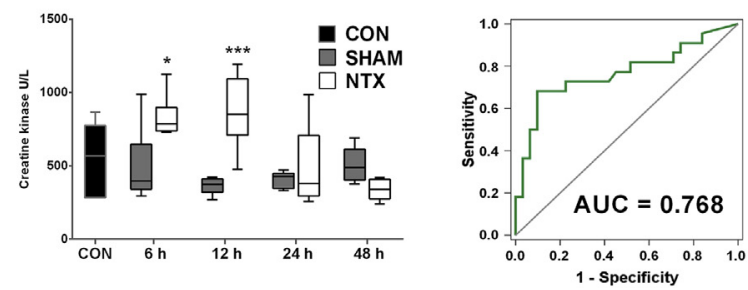

ajp.amjpathol.org - The American Journal of Pathology 
heavy chain content was analyzed by SDS PAGE and silver staining as previously described. ${ }^{31}$ The second half was placed in $40 \mu \mathrm{L}$ of Buffer RLT (Qiagen) and stored at $-80^{\circ} \mathrm{C}$. Total RNA was extracted with QIAzol reagent (Qiagen) according to the manufacturer's protocol. cDNA was synthesized with a Universal cDNA Synthesis Kit (Exiqon) from $5 \mu \mathrm{L}$ of RNA (diluted 1:6) as described in cDNA Synthesis. PCR reactions were performed from $4 \mu \mathrm{L}$ of cDNA (diluted 1:80).

\section{Platform Concordance Validation}

cDNA was synthesized with the miScript II RT Kit (Qiagen) from $1 \mu \mathrm{L}$ of RNA from the NTX (12 hours) and CON groups from experiment 1 . Individual qPCR measurements were performed on a LightCycler 480 instrument (Roche Diagnostics), with miScript Primer Assays (Qiagen) in a $10-\mu \mathrm{L}$ reaction volume using $3 \mu \mathrm{L}$ of cDNA (diluted $1: 11$ ).

\section{Influence of Pre-Analytical Conditions (Experiment 5)}

For RBC enrichment, control blood samples were centrifuged ( $2000 \times g$ for 10 minutes), and $1 \mathrm{~mL}$ of RBCs were saved and washed in $9 \mathrm{~mL}$ of $\mathrm{NaCl}$. After centrifugation, $500 \mu \mathrm{L}$ of $\mathrm{RBCs}$ were frozen $\left(-80^{\circ} \mathrm{C}\right)$, thawed, and vortexed for 1 minute. RBC lysate was used to enrich four control plasma pools to $1 \%$ of their total volume. The $1 \%$ enriched pools were diluted with clear plasma to achieve $0.5 \%, 0.25 \%, 0.125 \%$, and $0.0625 \%$ of RBC enrichment.

For platelet enrichment, $5 \mathrm{~mL}$ of control blood was transferred to a No Additive (Z) Plus Tube (BD Vacutainer; Becton, Dickinson and Company) with $555 \mu \mathrm{L}$ of acidcitrate-dextrose buffer and $4 \mu \mathrm{L}$ of prostaglandin $\mathrm{E}_{1}$ (SigmaAldrich). After centrifugation $(150 \times g$ for 20 minutes at room temperature), $500 \mu \mathrm{L}$ of supernatant was transferred to a new vial and $50 \mu \mathrm{L}$ of acid-citrate-dextrose buffer was added. Samples were centrifuged $(900 \times g$ for 5 minutes at room temperature) and $30 \mu \mathrm{L}$ of the platelet-enriched pellets were collected, diluted in $170 \mu \mathrm{L}$ of $1 \mathrm{X}$ phosphate-buffered saline, and frozen $\left(-80^{\circ} \mathrm{C}\right)$. Six control plasma pools were enriched with lysate to $4 \%$ of their volume, and diluted to achieve $1 \%, 0.5 \%$, and $0.25 \%$ of platelet enrichment.

For extracellular vesicle isolation, $100 \mu \mathrm{L}$ of thromboplastin D (SBI System Biosciences, Mountain View, CA) and $100 \mu \mathrm{L}$ of plasma pools were mixed and incubated for 15 minutes at $37^{\circ} \mathrm{C}$. Samples were centrifuged $(10,000 \times g$ for 5 minutes at room temperature). Then, $50 \mu \mathrm{L}$ of $1 \mathrm{X}$ phosphate-buffered saline was added. Extracellular vesicles were isolated using Total Exosome Isolation Reagent (InvitroGen, Carlsbad, CA). For all protocols, total RNA extraction, reverse transcription, and individual qPCR miRNA downstream measurements were performed as previously described in Plasma miRNA Extraction, cDNA Synthesis, and Circulating miRNA Profiling (Experiment 1).

\section{Statistical Analysis}

The results are represented as box-and-whiskers plots or as scatter dot plots. Graphics were generated using Prism software version 6.01 (GraphPad, San Diego, CA). Statistical tests were performed on $\log _{10}$-transformed data with Statistica software version 10 (StatSoft, Maison-Alfort, France). The results of experiment 1 were analyzed with a two-way analysis of variance to evaluate global effects of condition and time. Receiver operating characteristic curve analysis was performed with IBM SPSS Statistics version 20 (IBM Corp., Armonk, NY). The results of experiments 2 to 4 were analyzed with a one-way analysis of variance. The Bonferroni post hoc test was used for intergroup comparisons, when appropriate. The comparison of data from the CON and NTX groups at 12 hours (for platform concordance validation) was performed using a $t$-test. When $n$ was $<8$, nonparametric tests were used. Fiber phenotype and RBC- and platelet- enrichment results were analyzed with a nonparametric Kruskal-Wallis test. For analyses of vesicular and nonvesicular fractions in CON compared to NTX at 12 hours, intramuscular miRNA content of damaged and nondamaged muscle were compared with a nonparametric $U$-test. Results with a $P$ value $\leq 0.05$ were considered significant.

\section{Results}

\section{Circulating MyomiRs Are Affected by Skeletal Muscle Damage}

Notexin-induced damage was extensive, as shown by histological examination of hematoxylin phloxine saffron-stained cryosections, with necrotic muscle fibers found as soon as 6 hours after injection and almost no intact myofiber at 48 hours. Infiltrating mononuclear cells were present 6 hours after injection and later, surrounding fibers or inside necrotic cells

\footnotetext{
Figure 1 Muscle histological examination and plasma miRNA measurement after soleus muscle damage. Cryosections were stained with hematoxylin phloxine saffron. A-D: Control muscle (A) and muscle damage at 6 (B), 12 (C), and 48 (D) hours after notexin injection. B and C: Extensive muscle damage shows as pale necrotic myofibers and infiltration of mononuclear cells. D: At 48 hours, almost no intact fibers remain. $\mathbf{E}-\mathbf{N}$ : Muscle-specific miRNAs (E-J), Rattus norvegicus (rno)-miR-378a-3p (K), rno-miR-434-3p (L), rno-miR-136-5p (M), and creatine kinase (N) were measured in the plasma of control rats (CON); at $6,12,24$, and 48 hours after muscle damage (NTX); or after sham operation (SHAM). miRNAs were measured by real-time quantitative RT-PCR. Results were normalized with seven internal reference miRNAs. Receiver operating characteristic curve analyses were performed based on values at 6, 12, and 24 hours; NTX values were considered positive for muscle damage, CON and SHAM were considered negative. Data are expressed as interquartile ranges (boxes), medians (horizontal bars), and 5th and 95th percentiles (whiskers); corresponding area under the curve (AUC) values are given. $n=8$ rats per group per time point. ${ }^{*} P \leq 0.05,{ }^{* *} P \leq 0.01$, and ${ }^{* * *} P \leq 0.001$ versus SHAM at the same time point, as determined by a two-way analysis of variance with a subsequent Bonferroni post hoc test; complete main effect results are given in Supplemental Table S2. AU, arbitrary units; mmu, Mus musculus.
} 
(Figure 1). Plasma CK activity was increased, with a peak value at 12 hours and no effect of sham operation (Figure 1).

As previously reported in humans, miR-208b-3p and $-499-5 p$ were detected at very low levels and were often undetectable in the plasma of control and sham-operated rats (Figure 1). ${ }^{25,26}$ The myomiRs miR-1-3p, -133a-3p, -133b$3 p,-206-3 p,-208 b-3 p$, and $-499-5 p$ were substantially increased, and no effect of sham operation was found, except for miR-206-3p that slightly increased in SHAM at 12,24 , and 48 hours compared to $\mathrm{CON}$.

A study of the time course of miRNA response after notexin injection showed that plasma levels of musclespecific miRNAs peaked at 12 hours, except that of miR133a-3p; all reached baseline levels after 24 hours, except for miR-208b-3p (Figure 1 and Supplemental Table S2). Fold-changes compared those in the SHAM groups ranged from 10.5 for miR-499-5p to about 145 for miR-208b-3p.

Because notexin induces sarcolemmal lesions, ${ }^{32}$ we hypothesized that high plasma miRNA levels result from a massive leak from damaged fibers, and we measured myomiR levels within skeletal muscle in CON and NTX rats (12 hours after injection). Consistently, we found substantial decreases in myomiRs (Supplemental Figure S1).

The diagnostic accuracy of each individual miRNA was assessed by a receiver operating characteristic curve analysis (Figure 1). Because all myomiRs returned to control values by 48 hours after notexin injection, analysis of the receiver operating characteristic curve was performed based on values at 6,12 , and 24 hours. NTX rats were considered positive for muscle damage; the SHAM and CON groups were considered negative. Plasma myomiRs were able to discriminate positive and negative animals with very good specificity and sensitivity, as shown by high area under the curve (AUC) values (Figure 1). Plasma miR-206-3p and miR-499-5p had the lower AUC (0.871) and miR-208b-3p had the higher AUC (0.969), meaning that this miRNA could discriminate animals with muscle damage from those with almost no error. Noteworthy, CK accuracy was lower than that of any miRNA, with an AUC of 0.768 .

qPCR primer design for miRNAs is challenging due to their short length, close similarity, and the necessity to elongate the cDNA during reverse transcription. Hence, we validated the 12-hour sample results with another PCR system (Qiagen). These findings were similar between all damage-responsive miRNAs, indicating that they are system independent (data not shown). Similarly, we validated our results using cardiotoxin rather than notexin; all miRNAs were up-regulated with induced muscle damage, indicating that the response is not molecule dependent (data not shown).

\section{Two Non-Muscle-Specific miRNAs Have Profiles Similar to Those of MyomiRs}

Based on the profiling, non-muscle-specific miRNA were selected. Two of them, namely miR-378a-3p and -434-3p, revealed plasma profiles that were very similar to those of
myomiRs (Figure 1). The main difference with myomiRs was that the fold-changes were lower (3.52 and 4.19 for miR-378a-3p and -434-3p, respectively), with no effect of sham operation. As for myomiRs, whether plasma miRNA levels result from a leak from damaged muscle was assessed. miR-378a-3p was expressed in skeletal muscle of $\mathrm{CON}$ rats but did not significantly decrease in response to injury (Supplemental Figure S1). The overall evolution of miR-434 differed from those of myomiRs in two aspects: its concentration was still significantly higher in plasma (3.59-fold) than were CON values at 24 hours after notexin injection, and miR-434-3p levels remained unchanged in damaged muscle (Supplemental Figure S1), raising the question of its cellular origin in plasma. Taken together, both miR-378a-3p and -434-3p appear to be interesting circulating biomarkers of muscle damage, as shown by the receiver operating characteristic curve analysis, with AUCs of 0.889 and 0.900 for $\mathrm{miR}-378 \mathrm{a}-3 \mathrm{p}$ and $-434-3 p$, respectively (Figure 1)

The Dlk1-Dio3 cluster member miR-136-5p evolution was the same as those of myomiRs in response to injury (Figure 1), but sham operation induced an increase in miR136-5p at 6 hours after surgery, making the interpretation of any result difficult at that time point. This finding is illustrated by the receiver operating characteristic curve analysis, showing an AUC of 0.690 (Figure 1). Therefore, miR-136$5 p$ could be responsive to many stimuli, thereby compromising its utility as a biomarker.

\section{A Set of miRNAs Are Not Specifically Altered in Response to Muscle Damage}

Among the 72 miRNAs selected by our profiling, some were not specifically altered in the plasma of NTX rats. For 35 of them, we found no significant alterations in plasma levels. Among them, the cardiac-specific miR-208a-3p was expressed at very low levels and remained undetectable in most samples.

\section{Both Vesicular and Nonvesicular miRNA Fractions Increase after Muscle Damage}

Because skeletal muscle can secrete myomiR-containing microvesicles, ${ }^{33}$ we evaluated whether measuring miRNAs in extracellular vesicles could improve diagnosis, as proposed for liver injury. ${ }^{34} \mathrm{We}$ found significant rises in miR-1-3p, $-133 a-3 p,-133 b-3 p$, and $-206-3 p$ in the vesicular fraction (Figure 2A), whereas miR-208b-3p, -434-3p, and -499-5p remained undetectable in most samples (Figure 2A). Conversely, miR-378a-3p remained unchanged in the vesicular fraction after muscle damage (Figure 2A). In the nonvesicular fraction of plasma, increases were found in miR-1-3p, -133a-3p, and -206-3p (Figure 2B). miR-208b$3 p,-499-5 p$, and $-434-3 p$ were detectable and rose in the nonvesicular fraction after damage (Figure 2B). No alteration in miR-378a-3p was found (Figure 2B). Taken together, the 

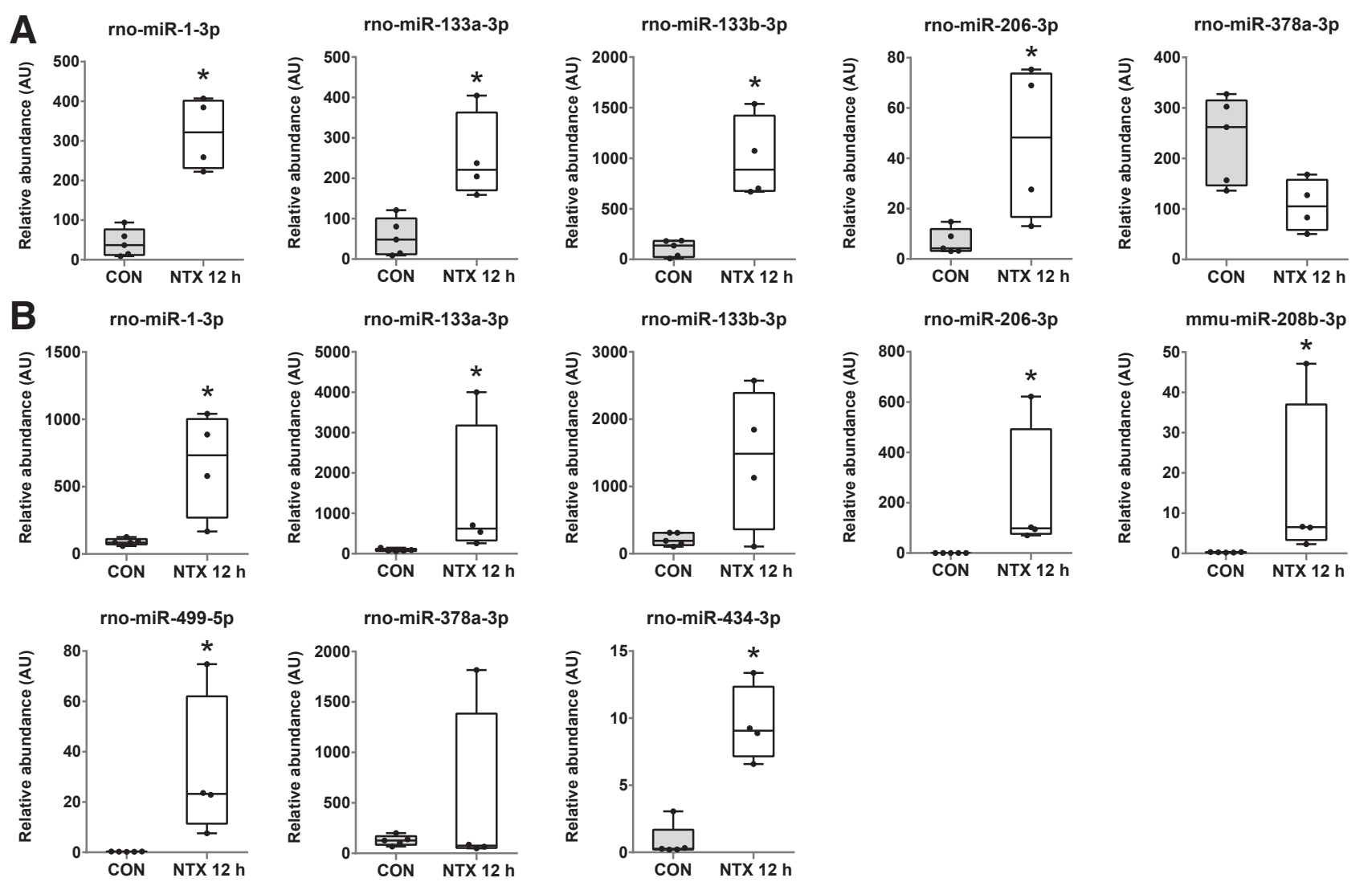

Figure 2 Damage-responsive miRNAs increase in both vesicular and nonvesicular fractions. A and B: Damage-responsive miRNAs were measured in plasma vesicular (A) and nonvesicular (B) fractions. Fractions were separated using the Total Exosome Isolation Kit (InvitroGen, Carlsbad, CA) from control plasma (CON) or from plasma 12 hours after notexin-induced muscle damage (NTX). miRNAs were measured by real-time quantitative RT-PCR. Results were normalized to five or three internal reference miRNAs for nonvesicular and vesicular results, respectively. miRNAs Mus musculus (mmu)-miR-208b-3p, Rattus norvegicus (rno)-miR-499-5p, and rno-miR-434-3p were not detectable in the vesicular fraction. Data are expressed as interquartile ranges (boxes), medians (horizontal bars), 5 th and 95 th percentiles (whiskers), and individual values (dots). $n=4$ per fraction (NTX); $n=5$ per fraction (CON). ${ }^{*} P \leq 0.05$ versus CON, as determined by a $U$-test. $\mathrm{AU}$, relative abundance arbitrary units.

results indicate that, depending on the miRNA concerned, both vesicular and nonvesicular fractions increase. Because separating these two fractions reduces the amount of RNA available for the RT-PCR process, and because the biomarkers we selected are not restricted to the vesicular fractions, we conclude that working on whole plasma samples remains the best option.

\section{Circulating miRNA Response to Muscle Damage Is Independent of Sex and Age}

Sex and age could be sources of interindividual variability both at baseline and in response to muscle damage. Baseline levels of selected miRNAs were similar in young male, young female, and aged male control rats, except for plasma miR-434-3p, which was undetectable in most female and aged rats. After notexin injection, comparable fold-changes were found in females (ranging from 4-fold for miR-378a$3 p$ to 43.63 -fold for miR-208b-3p compared to those in F-SHAM) (Figure 3A) and lower fold-changes were found in aged rats (ranging from 2.2-fold for miR-1-3p to 19.36-fold for miR-208b-3p compared with those in
A-SHAM) (Figure 3B). miR-378a did not increase after muscle damage (Figure 3B), and miR-434-3p remained undetectable in A-CON, A-SHAM, as well as A-NTX.

\section{Influence of Muscle Phenotype on Circulating miRNAs}

Adult skeletal muscle fibers are classified into slow- and fast-type categories according to their myosin heavy chain content. In rodents, myosin heavy chain I is the slow isoform; myosin heavy chain IIa, IIx, and IIb are the fast isoforms; and miRNAs are differentially expressed in slow- and fast-type muscles. ${ }^{35-37}$ This differential expression could result in different circulating profiles in response to slow- or fast-type muscle damage. We first studied miRNA expression in single muscle fibers. A subset of miRNAs were equally expressed in all fiber types, including miR-133a-3p and $-133 b-3 p$ (Figure 4). According to geNorm, miR-434-3p and $-378 a-3 p$ were the most stable and served as references. Therefore, no graphs are shown, but their expression levels were similar in all fiber types. Both miR-208b-3p and -499-5p were mainly expressed in type I fibers (Figure 4), consistent with 
A

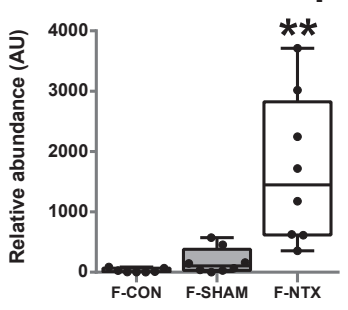

rno-miR-133b-3p

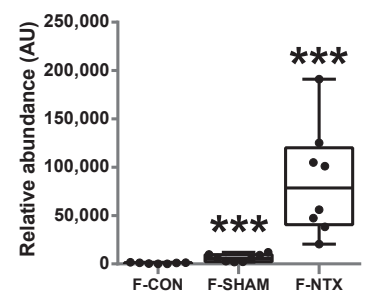

mmu-miR-208b

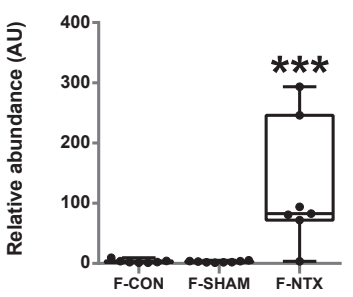

rno-miR-378a-3p

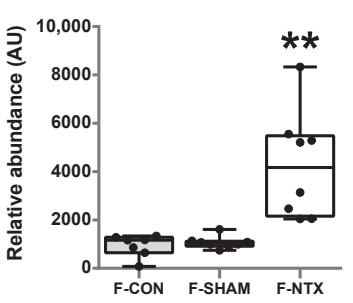

rno-miR-133a-3p

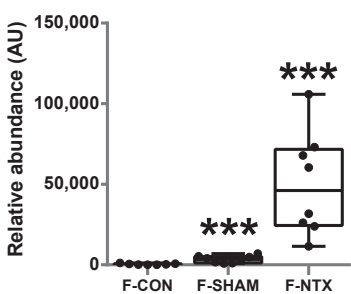

rno-miR-206-3p

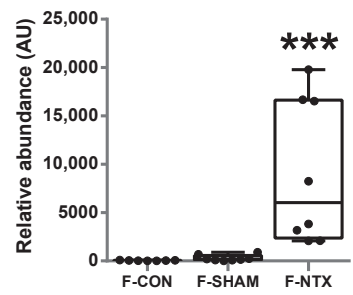

rno-miR-499-5p

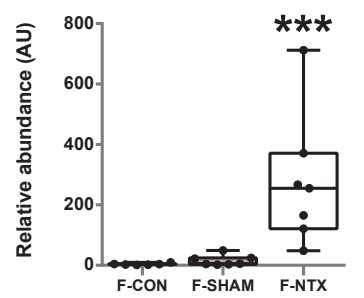

rno-miR-434-3p

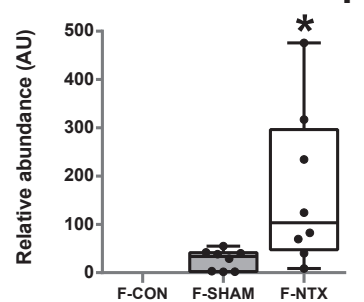

B rno-miR-1-3p

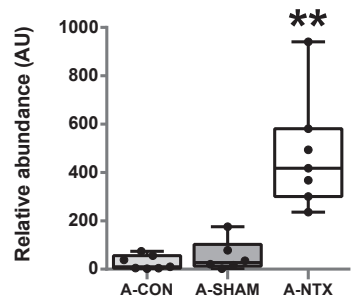

rno-miR-133b-3p

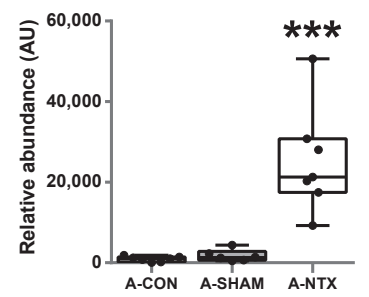

mmu-miR-208b-3p

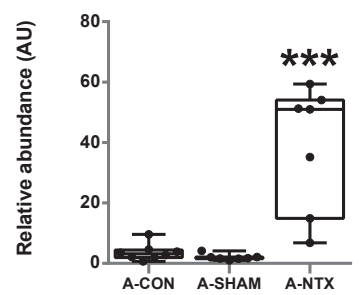

rno-miR-378a-3p

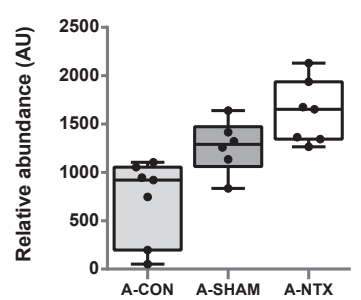

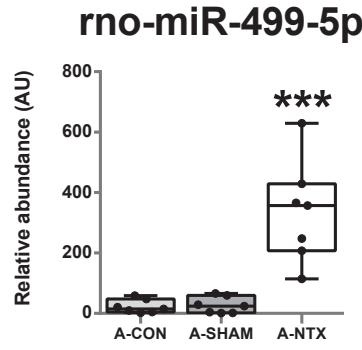

rno-miR-133a-3p

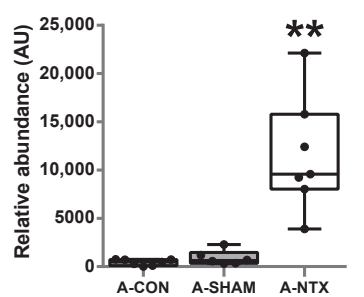

rno-miR-206-3p

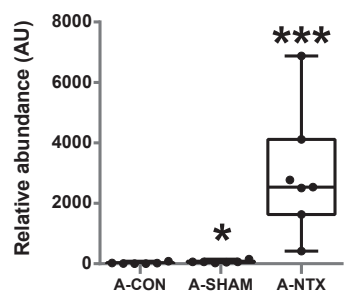

rno-miR-499-5p

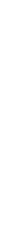

Figure 3 Influence of sex and age on damage-responsive miRNA levels. A and B: Damage-responsive miRNA levels were measured in the plasma of female (A) or aged (B) control rats (F-CON; A-CON), or 12 hours after sham operation (F-SHAM; A-SHAM) or notexin-induced muscle damage (F-NTX; A-NTX). miRNAs were measured by real-time quantitative RT-PCR. Results were normalized to seven or five internal reference miRNAs for female and aged rats, respectively. Data are expressed as interquartile ranges (boxes), medians (horizontal bars), 5th and 95th percentiles (whiskers), and individual values (dots). $n=8$ rats per group. ${ }^{*} P \leq 0.05,{ }^{* *} P \leq 0.01$, and ${ }^{* *} P \leq 0.001$ versus F-SHAM, as determined by a one-way analysis of variance with subsequent Bonferroni post hoc test. $\mathrm{AU}$, relative abundance arbitrary units; mmu, Mus musculus; rno, Rattus norvegicus.

the fact they are encoded in introns of slow-type genes, $M y h 7$ and $M y h 7 b$, respectively. ${ }^{38}$ miR-206-3p was expressed in all fiber types, with higher levels in types I, IIa, and IIa/IIx fibers (Figure 4). Conversely, higher expression of miR-1-3p was found in types IIb and IIx fibers (Figure 4).

To determine whether such differences in expression patterns can result in different circulating profiles, we measured plasma miRNA levels in response to fast-type muscle (EDL) damage and compared them to slow-type muscle (soleus) results. The histological examination of hematoxylin phloxine saffron-stained EDL cryosections showed similar muscle damage as observed in soleus muscle (data not shown). After muscle damage (12 hours), miR-133a-3p, -133b-3p, and -206-3p plasma responses were consistent with fiber-type distribution (Figure 5), with no significant differences in plasma levels between the EDL and soleus groups. Similar results were found for miR-378a$3 p$ and $-434-3 p$, which is in good agreement with the finding that they were among the most stable miRNAs. Plasma miR-206-3p levels were higher after soleus damage, in accordance with its fiber-type distribution (Figure 5). Conversely, miR-1-3p had an unexpected profile: Its level tended to be higher after soleus damage, an outcome that does not reflect the fiber-type distribution. Finally, the two myomiRs that showed high type I fiber specificity 

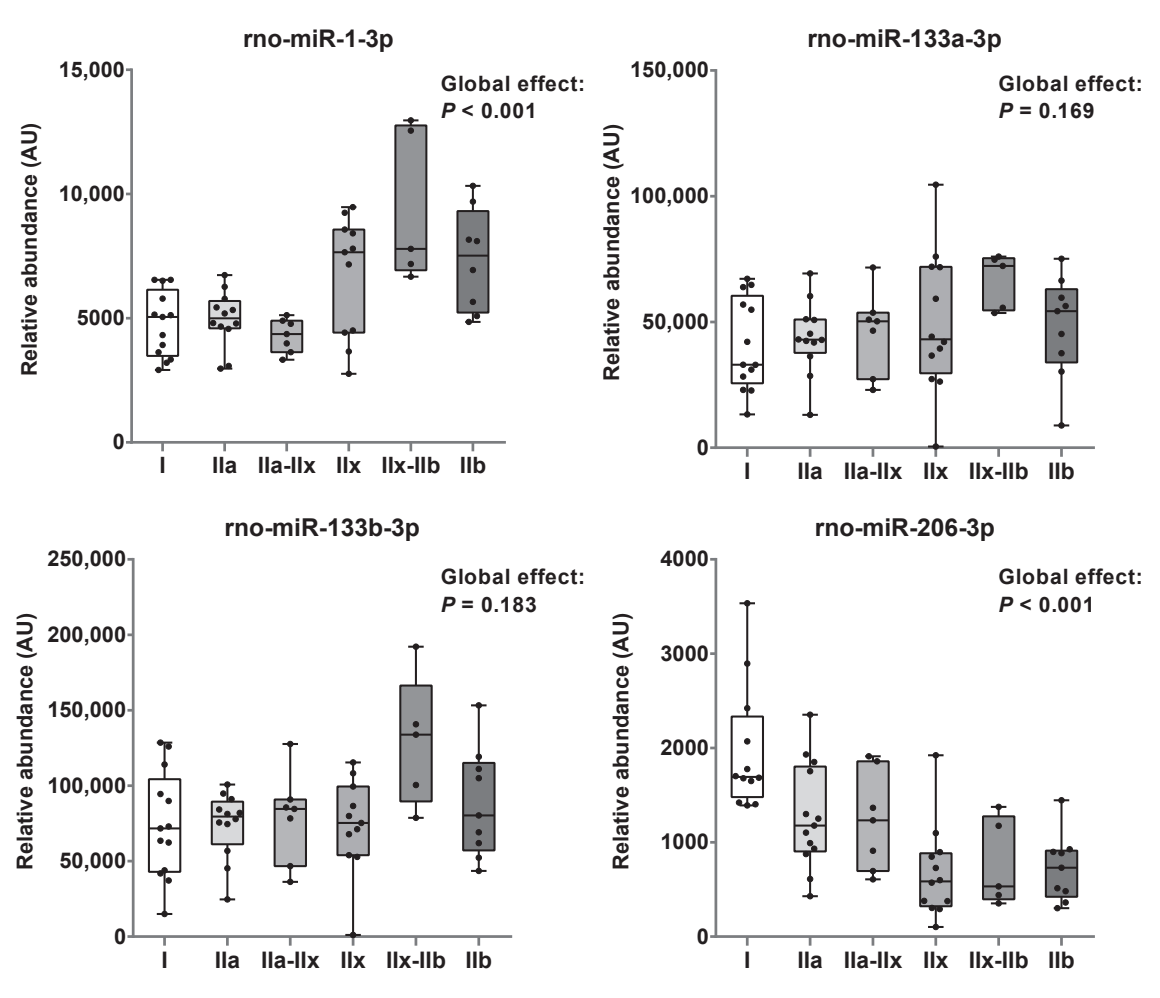

Figure 4 Damage-induced miRNA expression patterns in single muscle fibers. Single fibers were isolated from control plantaris muscles. One halffiber was used for determining myosin heavy chain isoforms and the other half-fiber was used for quantifying miRNAs. miRNA expressions were measured by real-time quantitative RT-PCR. Results were normalized to five internal reference miRNAs. The two non-muscle-specific miRNAs, Rattus norvegicus (rno)-miR-378a-3p and rno-miR434-3p, were selected by geNorm ${ }^{30}$ as stable reference miRNAs and were used to quantify the expression of the other miRNAs. Data are expressed as interquartile ranges (boxes), medians (horizontal bars), 5th and 95th percentiles (whiskers), and individual values (dots). $n=5$ (fiber type IIx-IIb); $n=7$ (fiber type IIa-IIx); $n=10$ (fiber type IIb); $n=13$ (fiber types I, IIa, and IIx). $P$ values are global effect results, as
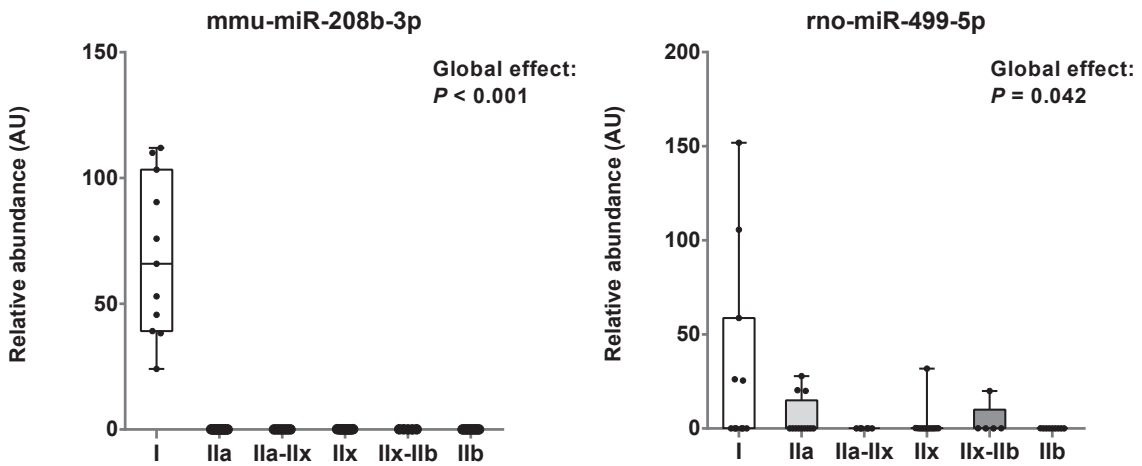
determined by a Kruskal-Wallis test. AU, relative abundance arbitrary units; mmu, Mus musculus.

(miR-208b-3p and -499-5p) had similar plasma responses in the EDL and soleus groups. Taken together, these observations indicate that, although the pattern of expression of miRNAs varies within myofibers, the overall impact of these differences on circulating profiles after acute damage seems to be very limited.

\section{Damage-Responsive Plasma miRNA Response Is Not Proportional to the Extent of Muscle Damage}

Ideally, a biomarker should indicate the extent of tissue damage. Therefore, whether the plasma miRNA response was proportional to the extent of muscle injury had to be determined. We injected notexin into two muscles (2 EDL) or four muscles ( $2 \mathrm{EDL}+2 \mathrm{TA})$, and compared the results to those in the EDL group studied in experiment 3 (1 EDL). Again, all measurements were performed in plasma collected 12 hours after damage. The damage-induced miRNAs were elevated in all groups compared with those in CON and SHAM. Unexpectedly, no significant differences were found between rats with one, two, or four muscles injured (Figure 6).

\section{Damage-Responsive miRNAs Are Not Affected by Pre-Analytical Conditions}

Pritchard et $\mathrm{al}^{39}$ showed that blood cells are major contributors to circulating miRNAs. Blood sample collection and processing can result in hemolysis or platelet contamination of plasma and can induce a substantial increase in various miRNAs. ${ }^{40-43}$ Therefore, from a biomarker perspective, it is necessary to study the effects of pre-analytical conditions. We first evaluated the impact of hemolysis by enriching plasma samples with increasing amounts of RBCs and measured damage-induced miRNAs. The optical deviation at $414 \mathrm{~nm}$ (free hemoglobin peak absorbance), as measured by spectrophotometry, was increased with hemolysis (data not shown), as was 

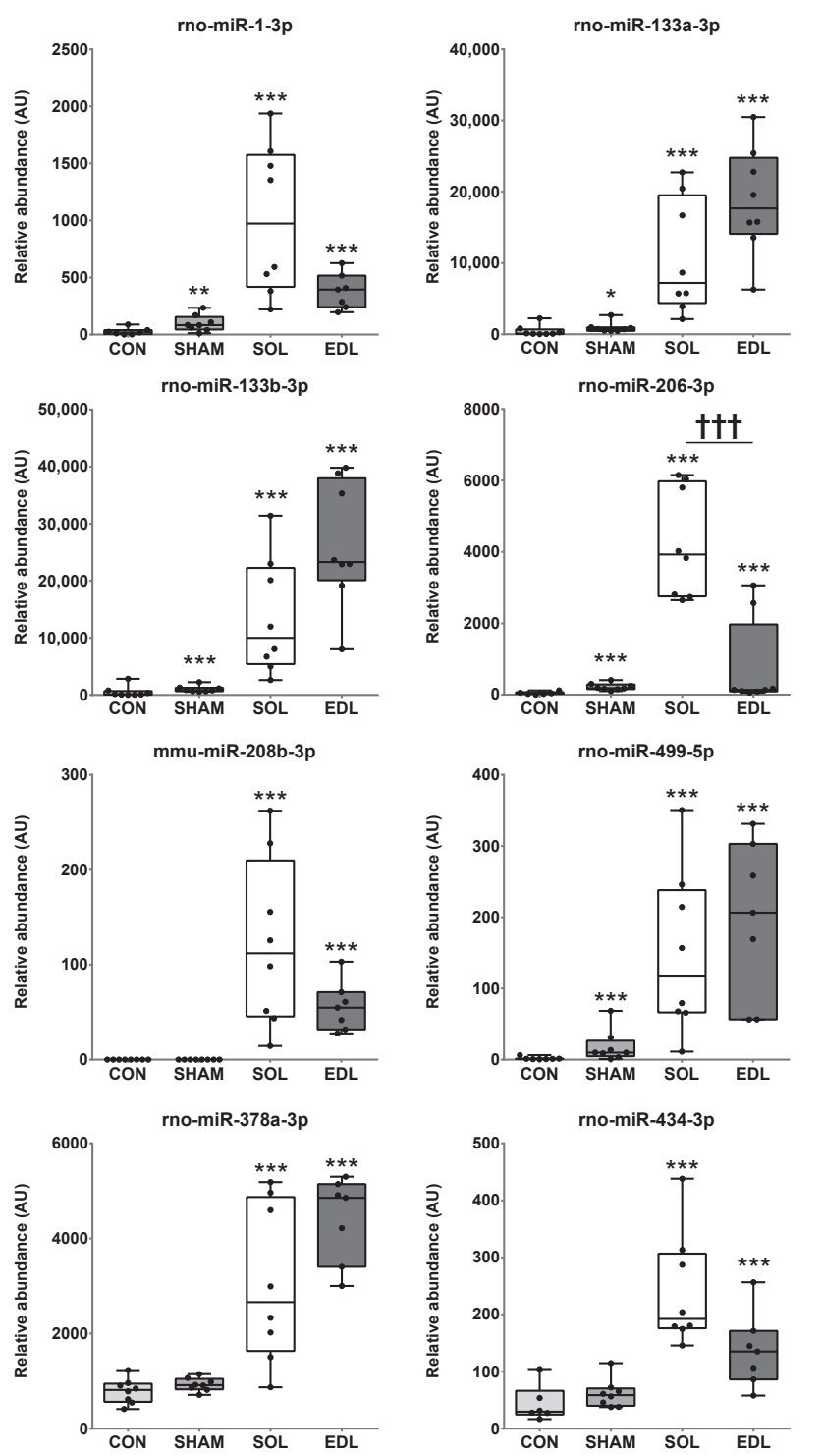

Figure 5 Damage-responsive plasma miRNAs in response to slow- or fasttype muscle damage. Damage of the slow-type soleus muscle (SOL) and the fast-type extensor digitorum longus (EDL) was induced by an intramuscular injection of notexin. Plasma miRNAs were measured by real-time quantitative RT-PCR in control rats (CON) and 12 hours after muscle damage or sham operation (SHAM). Results were normalized to six internal reference miRNAs. Data are expressed as interquartile ranges (boxes), medians (horizontal bars), 5th and 95th percentiles (whiskers), and individual values (dots). $n=8$ per group. ${ }^{*} P \leq 0.05,{ }^{* *} P \leq 0.01$, and ${ }^{* * *} P \leq 0.001$ versus $\mathrm{CON} ;{ }^{\dagger \dagger} P \leq 0.001$ for SOL versus EDL, as determined by a one-way analysis of variance analysis with subsequent Bonferroni post hoc test. AU, relative abundance arbitrary units; mmu, Mus musculus; rno, Rattus norvegicus.

RBC-enriched miR-451, as shown by decreasing qPCR quantification cycles (Figure 7A). Then, we measured the damage-responsive miRNAs and found that none of them was altered by hemolysis (Figure 7A). It should however be noted that miR-378a tended to increase with hemolysis $(P=0.073)$. Similarly, we enriched plasma samples with increasing amounts of platelets. As expected, the platelet-enriched levels of let-7f-5p and miR-103a-3p $p^{42}$ were increased, whereas the damage-responsive miRNAs were not affected (Figure 7B). Taken together, our results show that the miRNA set selected as candidate biomarkers of acute muscle injury are robust and are not influenced by pre-analytical RBC or platelet contamination of the plasma sample.

\section{Discussion}

Skeletal muscle damage is a very common pathologic event in healthy organisms. The clinical severity ranges from mild muscle pain or discomfort in those unaccustomed to exercise to life-threatening massive rhabdomyolysis. The clinical symptoms vary greatly with the extent of muscle damage and the etiology. Thus, diagnosis relies on laboratory tests. ${ }^{1}$ Serum CK activity is considered the most convenient and sensitive marker. It is routinely measured when rhabdomyolysis is suspected, for example, during treatment with potentially myotoxic drugs such as statins. ${ }^{44}$ However, the utilization of serum CK is sometimes difficult because of a high interindividual variability, ${ }^{45-48}$ as observed in our control group. Therefore, alternative and complementary biomarkers of muscle damage would be of great interest. Circulating miRNAs have emerged as reliable biomarkers in heart, liver, or pancreas injury. ${ }^{9,34,49}$ Here, we studied plasma miRNAs in response to acute muscle damage and found that a set of miRNAs, including both musclespecific and non-muscle-specific miRNAs, are robust and relevant biomarkers. Consistent with our previous work, ${ }^{25}$ we provide here results that support their utility as biomarkers of skeletal muscle damage, offering alternative solutions when CK interpretation is problematic.

Most myomiRs have been found to be reliable circulating biomarkers of muscular dystrophy in various animal models as well as in humans, leading to the concept of dystromiRs. ${ }^{20,21,50}$ It has been proposed that the dystromiRs released from dystrophic myofibers could be the result of an active release, possibly driven by local inflammatory factors. ${ }^{51}$ Yet, the underlying mechanisms remain largely unknown, and it is unclear whether they are markers of myofiber damage or of a degeneration-regeneration process. Our present results demonstrate that myomiRs respond to acute muscle damage in healthy organisms. Their substantial increase in plasma concomitant with their significant decrease in most muscles after notexin injection suggests that leaking through the damaged sarcolemma may be the main mechanism of release. Some damaged-induced miRNAs increase in the vesicular fraction of plasma, which may indicate an active release. Yet, these results could be interpreted as a nonspecific and passive release of the myofiber content, with miRNAs being within cytoplasmic microvesicles and others being nonencapsulated. ${ }^{52}$ However, an active release cannot be definitely excluded. Among the biomarker candidates, miR208b-3p and $-499-5 p$ are very interesting because they are barely detectable in noninjured rats, as was the case with $-208 \mathrm{~b}$ in humans and healthy dogs. ${ }^{21,25}$ Because miR-208b-3p and 

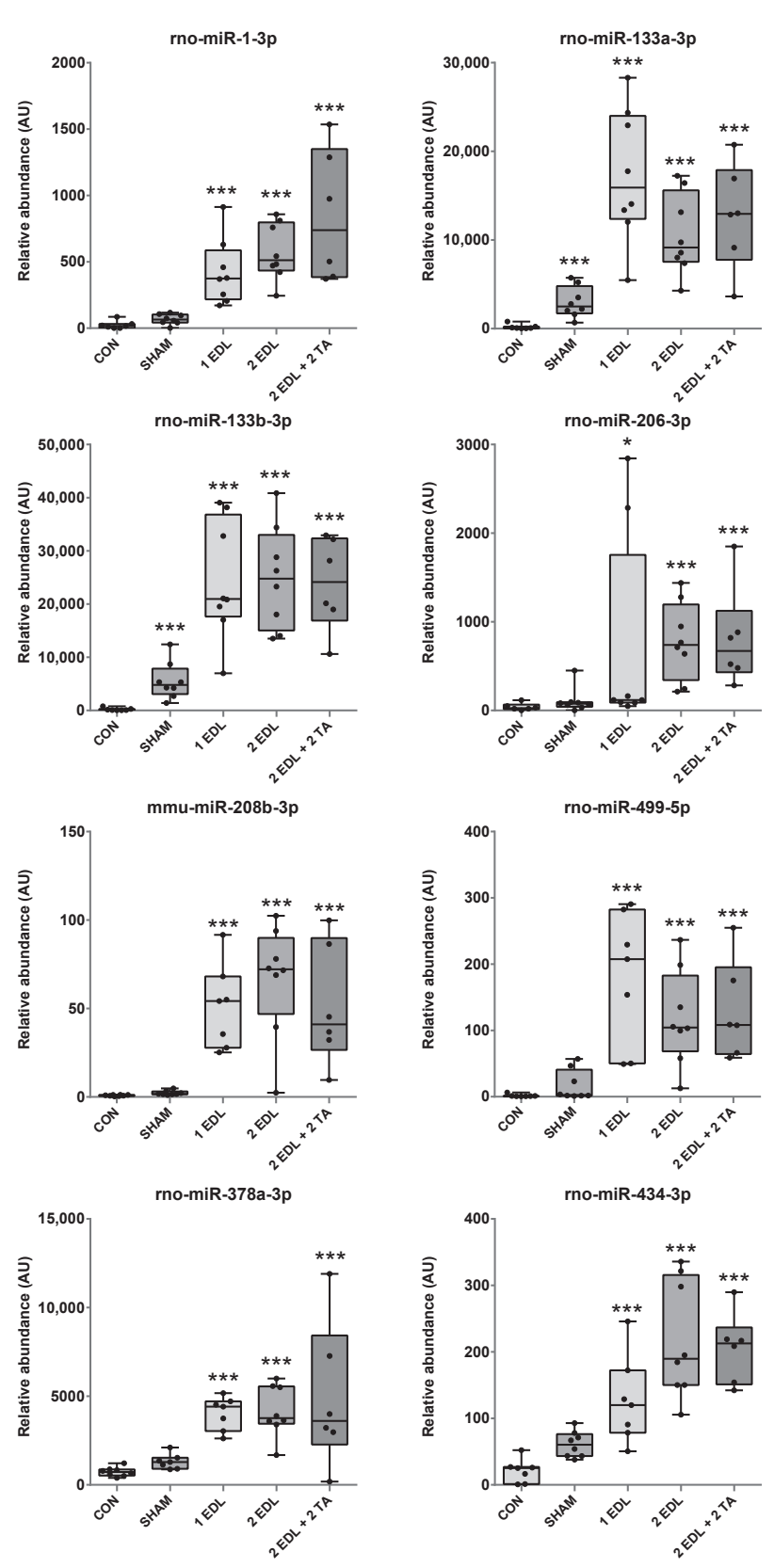

Figure 6 Damage-induced plasma miRNAs in response to increasing extent of muscle damage. Damage-induced miRNAs were measured in the plasma of control rats (CON) and sham-operated rats (SHAM) or 12 hours after an intramuscular injection of notexin in one or two extensor digitorum longus muscles (1 EDL; $2 \mathrm{EDL}$ ) or in two EDL and two tibialis anterior muscles (2 EDL $+2 \mathrm{TA})$. Plasma miRNAs were measured by real-time quantitative RT-PCR. Results were normalized to five internal reference miRNAs. Data are expressed as interquartile ranges (boxes), medians (horizontal bars), 5th and 95th percentiles (whiskers), and individual values (dots). $n=6$ (2 EDL +2 $\mathrm{TA}) ; n=8$ per group (CON, SHAM, $1 \mathrm{EDL}$, and $2 \mathrm{EDL}$ ). ${ }^{*} P \leq 0.05$, $* * * P \leq 0.001$ versus CON, as determined by a one-way analysis of variance analysis with subsequent Bonferroni post hoc test. $\mathrm{AU}$, relative abundance arbitrary units; mmu, Mus musculus; rno, Rattus norvegicus.

-499-5p have not been found in nonmuscle tissues, skeletal muscle could be the main contributor to their circulating form. These low baseline levels, together with a good responsiveness to muscle damage, may confer good sensitivity and specificity to these miRNAs. We also identified two non-muscle-specific miRNAs, miR-378a-3p and miR-434$3 p$, as biomarker candidates. Previous reports have shown that the miR-378 level was higher in plasma samples from three different mouse models of muscular dystrophies, as well as in dogs with golden retriever muscular dystrophy. ${ }^{20,21}$ In most of these models, muscular dystrophy is associated with a cardiomyopathy that contributes to alterations in circulating miRNA levels, including myomiRs, and alterations in miR-378 could not be attributed to muscle or cardiac dystrophy. Our results show a significant increase in plasma miR-378a-3p levels in the absence of cardiac damage. From a biomarker perspective, however, miR-378 should be interpreted with caution because plasma response is blunted in aged rats, and higher circulating levels are reported in colorectal, gastric, and renal cancers. ${ }^{53-55}$ miR-434-3p was not affected in the sera of sarcoglycan $\alpha$ or $\gamma$-null dystrophic mice; only a mild increase (2.4-fold) was reported in $m d x$ mice. Consequently, miR-434 was considered as a marker that could help in discriminating between pathologies rather than as a biomarker of dystrophy. ${ }^{20}$ We show that it is also an interesting marker of acute muscle damage in healthy organisms, except in aged rats. Surprisingly, no drop in miR434-3p was found in muscle; therefore, it is unclear whether the rise in plasma levels was due to a leak from damaged fibers or from another cell source. Whether early infiltrating cells such as neutrophils, monocytes, or macrophages can express miR434 is unknown. It is also worth noting that miR-434 is encoded within an intron of $R t l l$, a gene located on the reverse strand of the Dlk1-Dio3 locus. This genomic region contains a large miRNA cluster deregulated in the serum of dogs with golden retriever muscular dystrophy ${ }^{21}$ and found to be up-regulated in regenerating muscle. ${ }^{56}$ However, only one miRNA of the Dlkl-Dio3 megacluster was selected by our profiling (miR-136-3p), suggesting that miR-434 increases in response to muscle damage rather than muscle regeneration.

Blood biomarkers specific for damage in type I or II muscle fibers could be of interest in a research context, in which fiber-type differences in injury susceptibility might be expected. ${ }^{57}$ In the present work, we describe different expression patterns within muscle fibers, with miRNAs being equally expressed in all fibers, whereas others were expressed predominantly in type I or II fibers. Yet, no major difference in plasma profiles was found after slow- and fasttype muscle damage, suggesting that miRNAs are not predictive of the damaged fiber type. Nonetheless, in most clinical situations in which rhabdomyolysis is suspected, potentially all muscle fiber types are concerned, and specific biomarkers are not necessary and probably not desirable. Although the miRNA differences we described in myofibers do not result in similar circulating miRNA profile, their utilization as biomarkers of muscle damage is not undermined.

Damage-induced miRNAs are transiently elevated and rapidly cleared from plasma, with most miRNAs having reverted to control values 24 hours after injection. This finding is consistent with previous observations showing a 
A

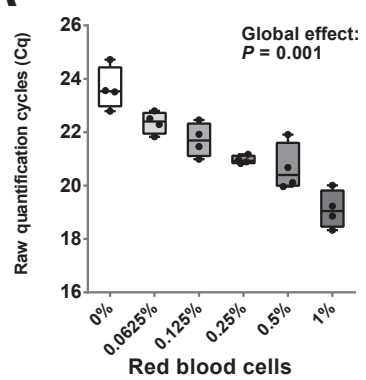

rno-miR-133a-3p

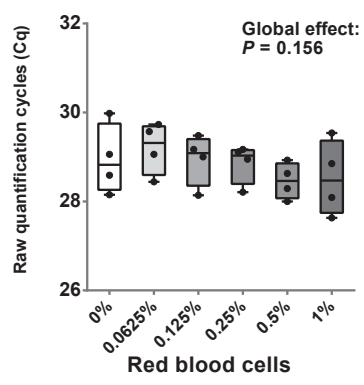

rno-miR-206-3p

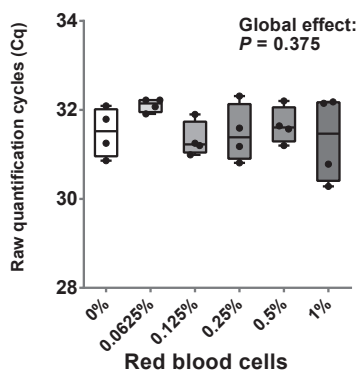

rno-miR-378a-3p

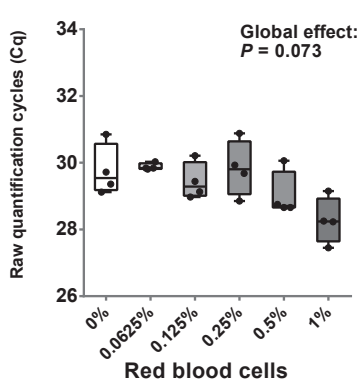

rno-miR-1-3p

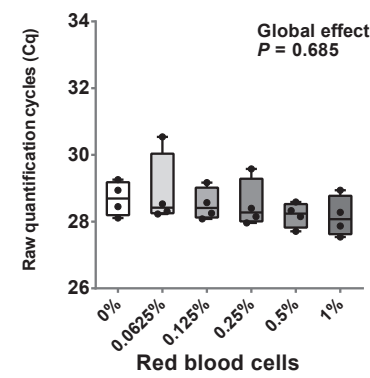

rno-miR-133b-3p

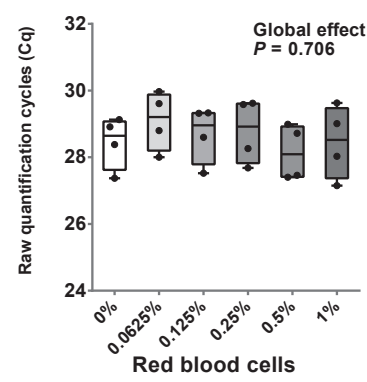

rno-miR-499-5p

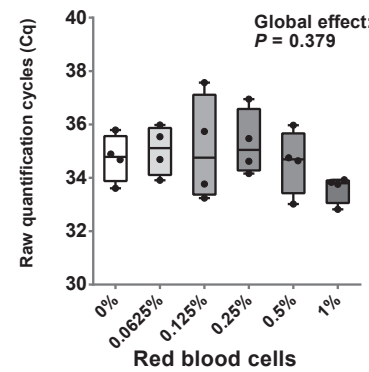

rno-miR-434-3p

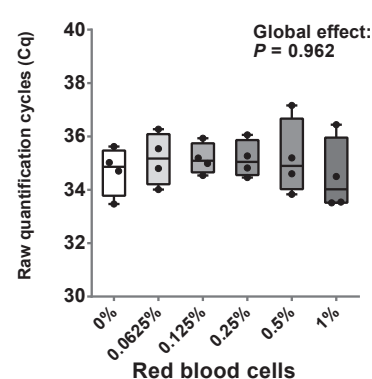

B

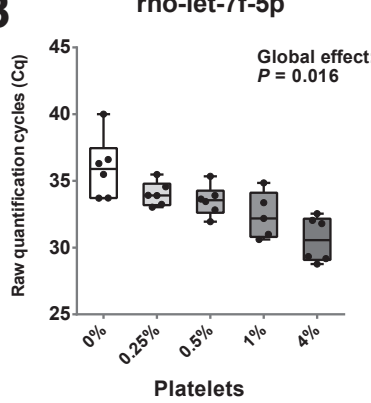

rno-miR-1-3p

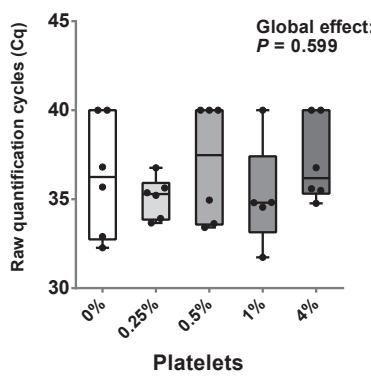

rno-miR-133b-3p

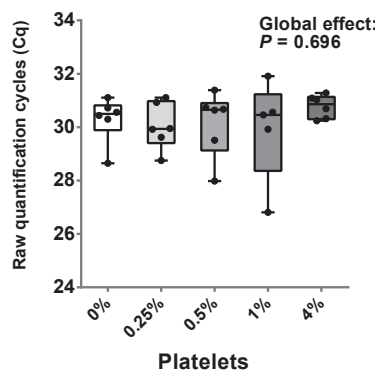

rno-miR-378a-3p

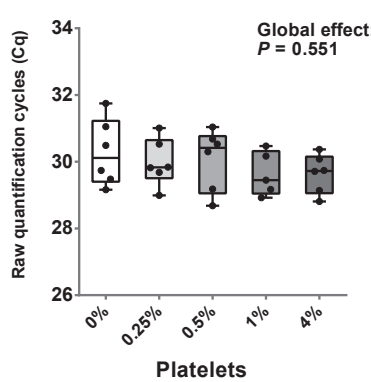

rno-miR-103-3p

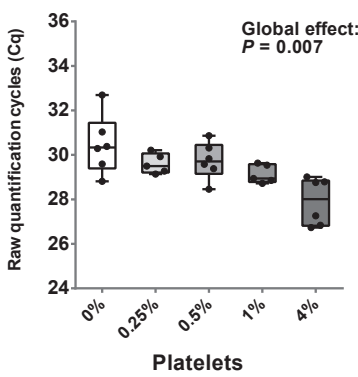

rno-miR-133a-3p

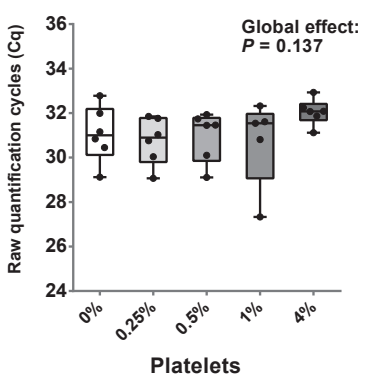

rno-miR-206-3p

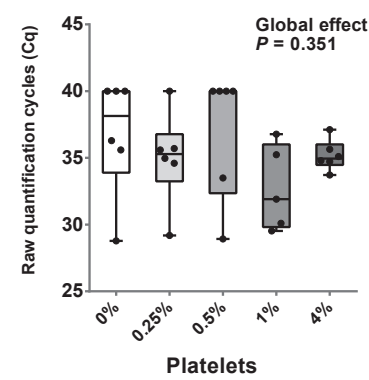

rno-miR-434-3p

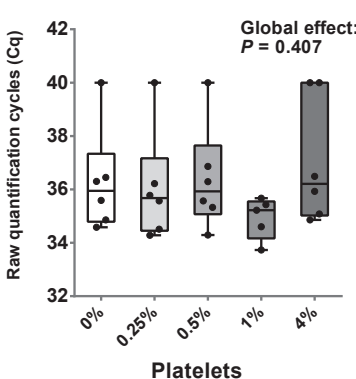

Figure 7 Damage-responsive plasma miRNAs and pre-analytical conditions. A and B: The impact of red blood cell (RBC) (A) or platelet (B) contamination on damage-responsive plasma miRNAs was evaluated. A: Control, nonhemolyzed plasma pools were enriched with RBCs to $1 \%$ of their total volume and progressively diluted to achieve $0.5 \%, 0.25 \%, 0.125 \%$, and $0.0625 \%$ of RBC enrichment. B: Control plasma pools were enriched with platelets to $4 \%$ of their total volume and progressively diluted to achieve $1 \%, 0.5 \%$, and $0.25 \%$ of platelet enrichment. The expressions of the RBC-enriched Rattus norvegicus (rno)miR-451-5p, the platelet-enriched rno-let-7f-5p and mo-miR-103-3p, and the damage-responsive miRNAs were measured by real-time quantitative RT-PCR. Low quantification cycle $(\mathrm{Cq})$ values indicate elevated miRNA abundance; high $\mathrm{Cq}$ values indicate low miRNA abundance. Data are expressed as interquartile ranges (boxes), medians (horizontal bars), 5th and 95th percentiles (whiskers), and individual values (dots) of raw Cq values. $n=4$ (A); $n=6$ (B). $P$ values are the global effect results, as determined by a Kruskal-Wallis test.

peak elevation of myomiRs in plasma 6 hours after acute eccentric exercise ${ }^{25}$ or 3 to 12 hours after experimental coronary artery ligation in rats. ${ }^{9}$ This rapid clearance is not a limitation because, contrary to our experimental model, pathologic processes, such as rhabdomyolysis or chronic myotoxic damage induced by statin treatment, do not affect myofibers at the same moment. Therefore, it does not compromise the utility of circulating miRNAs in the diagnosis of muscle damage and may even be of great interest because biomarkers can help to monitor the regression of an acute phenomenon. 
Very little is known about the biodistribution and clearance of circulating miRNAs. Recent work has suggested that circulating miRNAs could be trapped in the liver, adipose tissue, and lung, ${ }^{58}$ or eliminated in urine. ${ }^{59}$ An unexpected consequence of rapid plasma clearance could be the lack of correlation between plasma miRNAs and the extent of muscle damage in our study. It is unlikely that differences in miRNA release occurred within the different muscles (EDL and TA). Therefore, the similar levels of plasma miRNAs observed with one, two, or four muscles damaged may have been due to miRNA entrapment in tissues and renal clearance.

The ability of a biomarker to clearly discriminate between skeletal muscle-derived and cardiac-derived miRNAs is required in a context of muscle-damage diagnostics. miR-1, $-133 a,-208 b$, and $-499-5 p$ are enriched in both skeletal and cardiac muscle. They are increased in plasma in acute and chronic cardiac diseases such as myocardial infarction or heart failure. ${ }^{60}$ Thus, although they are very responsive to skeletal muscle damage, they are not fully specific. We systematically measured the cardiac-specific miR-208a-3p and found that it remained undetectable in most of our samples. This finding indicates that, as expected, no cardiac damage occurred and suggests that measuring miR-208a together with miR-1, -133a, and -499 can help to discriminate between a cardiac and a skeletal muscle origin. Two miRNAs, miR-133b and -206 , originating from the same bicistronic transcript, have been reported as specific of skeletal muscle ${ }^{61}$ and are increased in response to skeletal muscle damage. Because they have not been reported to increase in plasma after myocardial infarction, they are key biomarkers that should systematically be measured in association with other myomiRs. Then, although most miRNAs taken individually have shown very high diagnostic accuracy, the real strength of this approach in biomarker applications is the possibility of combining measurements to optimize diagnosis. Based on our results, we propose that measuring miR-208b-3p (best diagnostic accuracy) together with $-208 a-3 p$ (cardiac specific) and $-206-3 p$ or $-133 b-3 p$ (skeletal muscle specific) is a judicious combination.

\section{Conclusion}

We show that circulating miRNAs are interesting biomarkers of muscle damage in healthy individuals and that they could be a useful alternative to classic markers. This approach is promising and must be further developed before being usable in a clinical context. Major points have to be taken into consideration. First, the techniques we used are very reproducible and robust but time-consuming. Thus, automated steps such as high-quality RNA isolation systems have to be considered. Second, although guidelines have been proposed, no consensus exists on key points such as quality control or normalization of the results. New techniques based on probes rather than PCR may simplify and help to standardize the measurement of circulating miRNAs.
Finally, control values from healthy subjects have to be determined in large-scale cohorts to explore interindividual variability. Because circulating miRNAs have a high potential as biomarkers in many fields, new technical solutions are currently being developed to solve these problems and make miRNA measurements applicable in clinical laboratories.

\section{Acknowledgments}

We thank Hervé Chaussard, David Coulon, and Didier Lici for their help during animal studies; Julien Guéritée for English proofreading; and Drs. Patrick Saint-Blancard and Philippe Vest (Hôpital d'Instruction des Armées Percy) for their technical support.

\section{Supplemental Data}

Supplemental material for this article can be found at http://dx.doi.org/10.1016/j.ajpath.2016.01.007.

\section{References}

1. Khan FY: Rhabdomyolysis: a review of the literature. Neth J Med 2009, 67:272-283

2. Cervellin G, Comelli I, Lippi G: Rhabdomyolysis: historical background, clinical, diagnostic and therapeutic features. Clin Chem Lab Med 2010, 48:749-756

3. Brancaccio P, Maffulli N, Limongelli FM: Creatine kinase monitoring in sport medicine. Br Med Bull 2007, 81-82:209-230

4. Kanda K, Sugama K, Hayashida H, Sakuma J, Kawakami Y, Miura S, Yoshioka H, Mori Y, Suzuki K: Eccentric exercise-induced delayed-onset muscle soreness and changes in markers of muscle damage and inflammation. Exerc Immunol Rev 2013, 19:72-85

5. Ohlendieck K: Proteomic identification of biomarkers of skeletal muscle disorders. Biomark Med 2013, 7:169-186

6. Welsh M, Allen D, Byrnes W: Plasma matrix metalloproteinase-9 response to downhill running in humans. Int J Sports Med 2014, $35: 363-370$

7. Chang T-C, Mendell JT: microRNAs in Vertebrate Physiology and Human Disease. Annu Rev Genomics Hum Genet 2007, 8:215-239

8. Mitchell PS, Parkin RK, Kroh EM, Fritz BR, Wyman SK, PogosovaAgadjanyan EL, Peterson A, Noteboom J, O'Briant KC, Allen A, Lin DW, Urban N, Drescher CW, Knudsen BS, Stirewalt DL, Gentleman R, Vessella RL, Nelson PS, Martin DB, Tewari M: Circulating microRNAs as stable blood-based markers for cancer detection. Proc Natl Acad Sci U S A 2008, 105:10513-10518

9. Wang GK, Zhu JQ, Zhang JT, Li Q, Li Y, He J, Qin YW, Jing Q: Circulating microRNA: a novel potential biomarker for early diagnosis of acute myocardial infarction in humans. Eur Heart J 2010, 31: 659-666

10. Baggish AL, Hale A, Weiner RB, Lewis GD, Systrom D, Wang F, Wang TJ, Chan SY: Dynamic regulation of circulating microRNA during acute exhaustive exercise and sustained aerobic exercise training: circulating microRNA in exercise. J Physiol 2011, 589: 3983-3994

11. Baggish AL, Park J, Min P-K, Isaacs S, Parker BA, Thompson PD Troyanos C, D'Hemecourt P, Dyer S, Thiel M, Hale A, Chan SY: Rapid upregulation and clearance of distinct circulating microRNAs after prolonged aerobic exercise. J Appl Physiol 2014, 116:522-531

12. Laterza OF, Lim L, Garrett-Engele PW, Vlasakova K, Muniappa N, Tanaka WK, Johnson JM, Sina JF, Fare TL, Sistare FD, Glaab WE: 
Plasma MicroRNAs as Sensitive and Specific Biomarkers of Tissue Injury. Clin Chem 2009, 55:1977-1983

13. Cheng Y, Tan N, Yang J, Liu X, Cao X, He P, Dong X, Qin S, Zhang C: A translational study of circulating cell-free microRNA-1 in acute myocardial infarction. Clin Sci 2010, 119:87-95

14. D'Alessandra Y, Devanna P, Limana F, Straino S, Di Carlo A, Brambilla PG, Rubino M, Carena MC, Spazzafumo L, De Simone M, Micheli B, Biglioli P, Achilli F, Martelli F, Maggiolini S, Marenzi G, Pompilio G, Capogrossi MC: Circulating microRNAs are new and sensitive biomarkers of myocardial infarction. Eur Heart J 2010, 31: 2765-2773

15. Kirby TJ, Chaillou T, McCarthy JJ: The role of microRNAs in skeletal muscle health and disease. Front Biosci (Landmark Ed) 2015, 20:37-77

16. Russell AP, Wada S, Vergani L, Hock MB, Lamon S, Léger B, Ushida T, Cartoni R, Wadley GD, Hespel P, Kralli A, Soraru G, Angelini C, Akimoto T: Disruption of skeletal muscle mitochondrial network genes and miRNAs in amyotrophic lateral sclerosis. Neurobiol Dis 2012, 49:107-117

17. Wang XH: MicroRNA in myogenesis and muscle atrophy. Curr Opin Clin Nutr Metab Care 2013, 16:258-266

18. Rao PK, Missiaglia E, Shields L, Hyde G, Yuan B, Shepherd CJ, Shipley J, Lodish HF: Distinct roles for miR-1 and miR-133a in the proliferation and differentiation of rhabdomyosarcoma cells. FASEB J 2010, 24:3427-3437

19. Cacchiarelli D, Legnini I, Martone J, Cazzella V, D’Amico A, Bertini E, Bozzoni I: miRNAs as serum biomarkers for Duchenne muscular dystrophy. EMBO Mol Med 2011, 3:258-265

20. Vignier N, Amor F, Fogel P, Duvallet A, Poupiot J, Charrier S, Arock M, Montus M, Nelson I, Richard I, Carrier L, Servais L, Voit T, Bonne G, Israeli D: Distinctive serum miRNA profile in mouse models of striated muscular pathologies. PLoS One 2013, 8:e55281

21. Jeanson-Leh L, Lameth J, Krimi S, Buisset J, Amor F, Le Guiner C, Barthélémy I, Servais L, Blot S, Voit T, Israeli D: Serum profiling identifies novel muscle miRNA and cardiomyopathy-related miRNA biomarkers in Golden Retriever Muscular Dystrophy Dogs and Duchenne muscular dystrophy patients. Am J Pathol 2014, 184:2885-2898

22. Zaharieva IT, Calissano M, Scoto M, Preston M, Cirak S, Feng L, Collins J, Kole R, Guglieri M, Straub V, Bushby K, Ferlini A, Morgan JE, Muntoni F: Dystromirs as serum biomarkers for monitoring the disease severity in Duchenne muscular dystrophy. PLoS One 2013, 8:e80263

23. Perfetti A, Greco S, Bugiardini E, Cardani R, Gaia P, Gaetano C, Meola G, Martelli F: Plasma microRNAs as biomarkers for myotonic dystrophy type 1. Neuromuscul Disord 2014, 24:509-515

24. Roberts TC, Blomberg KEM, McClorey G, Andaloussi SE, Godfrey C, Betts C, Coursindel T, Gait MJ, Edvard Smith C, Wood MJ: Expression analysis in multiple muscle groups and serum reveals complexity in the microRNA transcriptome of the mdx mouse with implications for therapy. Mol Ther Nucleic Acids 2012, 1:e39

25. Banzet S, Chennaoui M, Girard O, Racinais S, Drogou C, Chalabi H, Koulmann N: Changes in circulating microRNAs levels with exercise modality. J Appl Physiol 2013, 115:1237-1244

26. Aoi W, Ichikawa H, Mune K, Tanimura Y, Mizushima K, Naito Y, Yoshikawa T: Muscle-enriched microRNA miR-486 decreases in circulation in response to exercise in young men. Front Physiol 2013, 4:80

27. Mooren FC, Viereck J, Kruger K, Thum T: Circulating microRNAs as potential biomarkers of aerobic exercise capacity. Am J Physiol Heart Circ Physiol 2013, 306:H557-H563

28. Witwer KW: Circulating microRNA biomarker studies: pitfalls and potential solutions. Clin Chem 2015, 61:56-63

29. De Guire V, Robitaille R, Tétreault N, Guérin R, Ménard C, Bambace N, Sapieha P: Circulating miRNAs as sensitive and specific biomarkers for the diagnosis and monitoring of human diseases: promises and challenges. Clin Biochem 2013, 46:846-860

30. Vandesompele J, De Preter K, Pattyn F, Poppe B, Van Roy N, De Paepe A, Speleman F: Accurate normalization of real-time quantitative RT-PCR data by geometric averaging of multiple internal control genes. Genome Biol 2002, 3. research0034

31. Banzet S, Koulmann N, Simler N, Birot O, Sanchez H, Chapot R, Peinnequin A, Bigard X: Fibre-type specificity of interleukin-6 gene transcription during muscle contraction in rat: association with calcineurin activity: fibre-type specificity of IL-6 gene transcription. J Physiol 2005, 566:839-847

32. Gutiérrez JM, Ownby CL: Skeletal muscle degeneration induced by venom phospholipases A2: insights into the mechanisms of local and systemic myotoxicity. Toxicon 2003, 42:915-931

33. Guescini M, Canonico B, Lucertini F, Maggio S, Annibalini G, Barbieri E, Luchetti F, Papa S, Stocchi V: Muscle releases AlphaSarcoglycan positive extracellular vesicles carrying miRNAs in the bloodstream. PLoS One 2015, 10:e0125094

34. Bala S, Petrasek J, Mundkur S, Catalano D, Levin I, Ward J, Alao H, Kodys K, Szabo G: Circulating microRNAs in exosomes indicate hepatocyte injury and inflammation in alcoholic, drug-induced, and inflammatory liver diseases. Hepatology 2012, 56:1946-1957

35. Muroya S, Taniguchi M, Shibata M, Oe M, Ojima K, Nakajima I, Chikuni K: Profiling of differentially expressed microRNA and the bioinformatic target gene analyses in bovine fast- and slow-type muscles by massively parallel sequencing. J Anim Sci 2013, 91:90-103

36. Liu Y, Li M, Ma J, Zhang J, Zhou C, Wang T, Gao X, Li X: Identification of differences in microRNA transcriptomes between porcine oxidative and glycolytic skeletal muscles. BMC Mol Biol 2013, $14: 7$

37. Allen DL, Loh AS: Posttranscriptional mechanisms involving microRNA-27a and b contribute to fast-specific and glucocorticoidmediated myostatin expression in skeletal muscle. Am J Physiol Cell Physiol 2011, 300:C124-C137

38. Van Rooij E, Quiat D, Johnson BA, Sutherland LB, Qi X, Richardson JA, Kelm RJ Jr, Olson EN: A family of microRNAs encoded by myosin genes governs myosin expression and muscle performance. Dev Cell 2009, 17:662-673

39. Pritchard CC, Kroh E, Wood B, Arroyo JD, Dougherty KJ, Miyaji MM, Tait JF, Tewari M: Blood Cell Origin of Circulating MicroRNAs: a cautionary note for cancer biomarker studies. Cancer Prev Res (Phila) 2012, 5:492-497

40. Kirschner MB, Kao SC, Edelman JJ, Armstrong NJ, Vallely MP, van Zandwijk N, Reid G: Haemolysis during sample preparation alters microRNA content of plasma. PLoS One 2011, 6:e24145

41. Cheng HH, Yi HS, Kim Y, Kroh EM, Chien JW, Eaton KD, Goodman MT, Tait JF, Tewari M, Pritchard CC: Plasma processing conditions substantially influence circulating microRNA biomarker levels. PLoS One 2013, 8:e64795

42. Plé H, Landry P, Benham A, Coarfa C, Gunaratne PH, Provost P: The repertoire and features of human platelet microRNAs. PLoS One 2012, 7:e50746

43. Willeit P, Zampetaki A, Dudek K, Kaudewitz D, King A, Kirkby NS, Crosby-Nwaobi R, Prokopi M, Drozdov I, Langley SR, Sivaprasad S, Markus HS, Mitchell JA, Warner TD, Kiechl S, Mayr M: Circulating microRNAs as novel biomarkers for platelet activation. Circ Res 2013, 112:595-600

44. Taha DA, De Moor CH, Barrett DA, Gershkovich P: Translational insight into statin-induced muscle toxicity: from cell culture to clinical studies. Transl Res 2014, 164:85-109

45. Nicholson GA, Morgan GJ, Meerkin M, Strauss ER, McLeod JG: The effect of aerobic exercise on serum creatine kinase activities. Muscle Nerve 1986, 9:820-824

46. Nicholson GA, Morgan G, Meerkin M, Strauss ER, McLeod JG: The creatine kinase reference interval: an assessment of intra-and interindividual Variation. J Neurol Sci 1985, 71:225-231

47. Brewster LM, Mairuhu G, Sturk A, van Montfrans GA: Distribution of creatine kinase in the general population: implications for statin therapy. Am Heart J 2007, 154:655-661

48. Kenney K, Landau ME, Gonzalez RS, Hundertmark J, O'Brien K, Campbell WW: Serum creatine kinase after exercise: drawing the line 
between physiological response and exertional rhabdomyolysis. Muscle Nerve 2012, 45:356-362

49. Goodwin D, Rosenzweig B, Zhang J, Xu L, Stewart S, Thompson K, Rouse R: Evaluation of miR-216a and miR-217 as potential biomarkers of acute pancreatic injury in rats and mice. Biomarkers 2014, 19:517-529

50. Mizuno H, Nakamura A, Aoki Y, Ito N, Kishi S, Yamamoto K, Sekiguchi M, Takeda S, Hashido K: Identification of muscle-specific microRNAs in serum of muscular dystrophy animal models: promising novel blood-based markers for muscular dystrophy. PLoS One 2011, 6:e18388

51. Li X, Li Y, Zhao L, Zhang D, Yao X, Zhang H, Wang Y, Wang X, Xia H, Yan J, Ying H: Circulating muscle-specific miRNAs in Duchenne Muscular Dystrophy patients. Mol Ther Nucleic Acids 2014, 3:e177

52. Chen X, Liang H, Zhang J, Zen K, Zhang CY: Secreted microRNAs: a new form of intercellular communication. Trends Cell Biol 2012, 22:125-132

53. Liu H, Zhu L, Liu B, Yang L, Meng X, Zhang W, Ma Y, Xiao H: Genome-wide microRNA profiles identify miR-378 as a serum biomarker for early detection of gastric cancer. Cancer Lett 2012, 316:196-203

54. Redova M, Poprach A, Nekvindova J, Iliev R, Radova L, Lakomy R, Svoboda M, Vyzula R, Slaby O: Circulating miR-378 and miR-451 in serum are potential biomarkers for renal cell carcinoma. J Transl Med 2012, 10:1479-5876
55. Zanutto S, Pizzamiglio S, Ghilotti M, Bertan C, Ravagnani F, Perrone F, Leo E, Pilotti S, Verderio P, Gariboldi M, Pierotti MA: Circulating miR-378 in plasma: a reliable, haemolysis-independent biomarker for colorectal cancer. Br J Cancer 2014, 110: 1001-1007

56. Snyder CM, Rice AL, Estrella NL, Held A, Kandarian SC, Naya FJ: MEF2A regulates the Gt12-Dio3 microRNA mega-cluster to modulate WNT signaling in skeletal muscle regeneration. Development 2013, 140:31-42

57. Carmona G, Guerrero M, Cussó R, Padullés JM, Moras G, Lloret M, Bedini JL, Cadefau JA: Muscle enzyme and fiber type-specific sarcomere protein increases in serum after inertial concentriceccentric exercise: serum sarcomere proteins increase by exercise. Scand J Med Sci Sports 2015, 25:e547-e557

58. Bala S, Csak T, Momen-Heravi F, Lippai D, Kodys K, Catalano D, Satishchandran A, Ambros V, Szabo G: Biodistribution and function of extracellular miRNA-155 in mice. Sci Rep 2015, 5:10721

59. Cheng Y, Wang X, Yang J, Duan X, Yao Y, Shi X, Chen Z, Fan Z, Liu X, Qin S, Tang X, Zhang C: A translational study of urine miRNAs in acute myocardial infarction. J Mol Cell Cardiol 2012, 53: 668-676

60. Sayed ASM, Xia K, Salma U, Yang T, Peng J: Diagnosis, Prognosis and Therapeutic Role of Circulating miRNAs in Cardiovascular Diseases. Heart Lung Circ 2014, 23:503-510

61. McCarthy J: MicroRNA-206: the skeletal muscle-specific myomiR. Biochim Biophys Acta 2008, 1779:682-691 